STUDI

FRANCESI

\section{Studi Francesi}

Rivista quadrimestrale fondata da Franco Simone

165 (LV | III) | 2011

LA RAPPRESENTAZIONE DELLA MADRE NELLA

LETTERATURA FRANCESE DEL NOVECENTO a cura di Dario Cecchetti e Michele Mastroianni

\title{
Alessandro Corio, Ilaria Vitali (dir.), Exilées, expatriées, nomades...
}

\section{Anna Zoppellari}

\section{(2) OpenEdition \\ Journals}

\section{Edizione digitale}

URL: http://journals.openedition.org/studifrancesi/5164

DOI: ERREUR PDO dans /localdata/www-bin/Core/Core/Db/Db.class.php L.34 : SQLSTATE[HY000]

[2006] MySQL server has gone away

ISSN: 2421-5856

Editore

Rosenberg \& Sellier

\section{Edizione cartacea}

Data di pubblicazione: 1 décembre 2011

Paginazione: 685-686

ISSN: 0039-2944

Notizia bibliografica digitale

Anna Zoppellari, «Alessandro Corio, Ilaria Vitali (dir.), Exilées, expatriées, nomades...», Studi Francesi [Online], 165 (LV | III) | 2011, online dal 30 novembre 2015, consultato il 07 janvier 2021. URL: http:// journals.openedition.org/studifrancesi/5164 ; DOI: https://doi.org/10.4000/studifrancesi.5164

Questo documento è stato generato automaticamente il 7 janvier 2021.

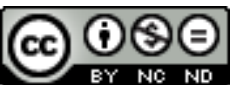

Studi Francesi è distribuita con Licenza Creative Commons Attribuzione - Non commerciale - Non opere derivate 4.0 Internazionale. 


\title{
Alessandro Corio, Ilaria Vitali (dir.), Exilées, expatriées, nomades...
}

\author{
Anna Zoppellari
}

\section{NOTIZIA}

ALESSANDRO CORIO, ILARIA VITALI (dir.), Exilées, expatriées, nomades..., «Francofonia», n. 58, 2010 , pp. 203.

1 Coordinato da Alessandro Corio e Ilaria Vitali, il n. 58 di «Francofonia» è dedicato alla scrittura femminile in situazione di erranza, esilio o comunque di allontanamento dal suolo natale e riunisce undici saggi dedicati a scrittrici non francesi che scrivono, in tutto o in parte, in francese.

2 Particolarmente interessante risulta il saggio introduttivo, in cui Corio e Vitali si interrogano sulle categorie critico-teoriche utilizzate nel panorama scientifico mondiale. Ricordando come il legame tra la scrittura e l'esilio sia costitutivo della letteratura stessa, i due studiosi si soffermano sulla accezione duplice, se non plurima, che esso assume nelle scrittrici migranti: se è vero che alla base di ogni scrittura è una «quête de soi qui doit passer inévitablement par un ailleurs [...] géographique, linguistique ou "intérieur"» (p.3), bisogna tuttavia ricordare che l'analisi dell'opera di una scrittrice exilée, implica necessariamente un'indagine sulla reciproca interdipendenza tra genere e allontanamento dal suolo natale. Scrivono, a questo proposito, i curatori: «l'écriture féminine est constitutivement une écriture de l'expatriation [...] par rapport à un espace établi et contrôlé par la "Loi du Père" ou de la "Patrie"» (p.3). Ne consegue una particolare propensione per la rilettura della norma, fosse anche quella che si è sviluppata all'interno delle scritture postcoloniali maschili. Esempio particolare di questo capovolgimento dei codici è l'intersezione tra discorso identitario e utilizzo della lingua francese. Laddove, nella parola maschile, identità e lingua di scrittura si inscrivono in una dinamica che, seppure non priva di sfaccettature, non prescinde mai dalla coscienza di utilizzare la lingua del colonizzatore 
(o dell'ex-colonizzatore), che è amata e odiata ad un tempo, nella parola femminile, ciò che risulta più significativo è il fatto che l'utilizzo del francese viene il più delle volte inteso come moto di accoglienza verso la lingua (e non contro o nonostante essa), in una sorta di «assimilation immunisante» (p.4) che tende ad accogliere l'altro attraverso una sostanziale riduzione del suo potere perturbante. Appoggiandosi all'elaborazione teorica di Hélène Cixous, i due studiosi recuperano il concetto di scrittura-corpo, che sfugge all'ideologia patriarcale e privilegia gli aspetti sensibili del linguaggio, siano essi sonori (fino al limite del silenzio) o visuali. La condizione di esclusa dalla letteratura acquisisce d'altra parte una valenza più drammatica e ambivalente in un contesto coloniale o postcoloniale, poiché «la subalterne-colonisée et femme-apparait doublement exclue et effacée des espaces sociaux» (p.5), come avviene in Assia Djebar, Calixthe Beyala, Fatou Diome e Ghania Hammadou. Il volume non ha tuttavia limitato l'analisi alle sole autrici riconducibili alla categoria della letteratura postcoloniale, ma si è inoltrato nel territorio più impervio della scrittura della migrazione e dell'esilio. È il caso delle scrittici rumene Rodica Iulian e Oana Orlea, della greca Mimika Kranaki, dell'ungherese Agota Kristof e della cinese Shan Sa, la cui opera è analizzata rispettivamente nei saggi di Elena-Brandusa Steiciuc, Vasiliki Lalagianni, Rachele Branchini e Emilie Guillerez. Per queste scrittrici, l'esperienza dell'esilio è sostanzialmente esperienza di dolore, tragedia e separazione, che viene ad un tempo curata e rivissuta attraverso la scrittura, in un perverso gioco di rievocazione dell'evento proprio nel momento in cui ci si vorrebbe curare la ferita.

Nello sforzo di trovare una chiave interpretativa che ad un tempo inglobi i diversi tipi di esperienza, ma garantisca le particolarità di ogni condizione, i due curatori hanno congegnato il volume con un occhio attento alle acquisizioni teoriche più recenti, ma sempre nella convinzione che ogni categoria costituisca una traccia e non una gabbia entro la quale chiudere il discorso analitico. Per questa via, Corio e Vitali riflettono sull'applicabilità dei concetti che vengono maggiormente utilizzati all'interno del panorama critico contemporaneo: astrazioni teorico-critiche quali quelle di esilio, espatrio, diaspora o scrittura migrante, costituiscono delle chiavi imprescindibili di introduzione ai testi, ma restano sostanzialmente «insuffisantes pour décrire les formes multiples et changeantes des itinéraires personnels et artistiques des écrivaines étudiées» (p. 7); elementi comuni da cui partire per uno sguardo d'insieme alla scrittura femminile delle exilées, expatriées e migrantes risultano peraltro i concetti di hybridité, ambivalenza, polifonia e creolizzazione che agiscono a livello delle singole opere, anch'essi tuttavia messi al vaglio delle diverse analisi. Corio e Vitali non dimenticano infine di interrogarsi sui molteplici tentativi di definizione che si sono succeduti nel corso dei decenni, siano essi mots-valises (enracinerrance, émigressance, migritude), nuove definizioni che partono dall'iscrizione dell'opera nella lingua e non nel territorio (littérature-monde) o studi che hanno analizzato il fenomeno migratorio a partire da una prospettiva transculturale e transnazionale.

4 Alla luce di queste considerazioni generali, è possibile ritrovare nei vari articoli le problematiche di fondo che riguardano la scrittura migrante e di genere in ambito francofono, quali la questione linguistico-identitaria, la funzione etica e le strategie estetiche della scrittura, i motivi dell'esilio e della memoria come elementi motori delle scelte tematiche e stilistiche. L'inscrizione delle opere all'interno della lingua francese, con tutto il suo carico di complessità esistenziale, caratterizza il saggio di Véronic Algeri dedicato alla perdita progressiva del francese in un contesto di arabizzazione diffusa: «dans La Disparition de la langue française, [Assia Djebar], sur le fonds du drame 
des disparus, impose la disparition de la langue du colonisateur et double cette perte par la disparition du protagoniste du roman» (p. 18). Ugualmente attraversato da un interesse metalinguistico è il saggio di Daniel S. Larangé su Calixthe Beyala: la scrittrice camerunense interpreta la ricerca identitaria come costituzione di un discorso francofono popolare non esente dalla pratica della citazione o del plagio. Particolarmente segnata da una preoccupazione stilistica e etica insieme è l'opera di Agota Kristof, in cui Rachele Branchini ha analizzato il lavoro di purificazione e cancellatura del superfluo attraverso l'analisi genetica e il confronto con i manoscritti.

Il motivo dell'esilio e quello delle strategie di rinascita in un'altra cultura sono reperibili nell'opera della scrittrice vietnamita Linda Lê (Julie ASSIER, Les migrances $d u$ moi: "Calomnies" de Linda Lê), della senegalese Fatou Diome, delle già citate Shan Sa, Mimika Kranaki, Rodica Iulian, Oana Orlea e della libanese Vénus Khoury-Ghata. Quest'ultima costituisce un caso assai particolare, poiché nella sua opera, l'esilio non si limita ad essere motivo attorno al quale la scrittrice intreccia il proprio discorso, ma costituisce un vero e proprio «laboratoire scripturaire» (p.163) in cui la lingua è pensata a partire da «un travail fécond qui sollicite l'invention et la créativité de l'écrivain, et qui fait qu'aucun de ses mots ne soit banal, ne serait-ce que parce que chaque phrase est le fruit d'une recherche langagière souvent absente dans la langue maternelle» (Ilaria Vitali, p. 155). Il riferimento all'allontanamento obbligato dalla terra madre ha esiti interessanti anche in Shan Sa, dove si radica nel tema del doppio (Emilie Guillerez), in Mimika Kranaki, in cui dà luogo ad una vera e propria esigenza di sopravvivenza della memoria (Vasiliki Lalagianni) o in Collette Fellous, scrittrice tunisina della comunità ebraica in cui Samia Kassab-Charfi ritrova la volontà di esprimere la migrazione attraverso una sorta di sovrapposizione geografica e linguistica. L'esigenza di trascrivere la condizione giovanile in un'Africa in via di trasformazione porta la senegalese Fatou Diome a rappresentare un mondo attento ai miti della cultura popolare e sportiva europea attraverso un dettato complesso che predilige il registro dell'oralità e utilizza la mescolanza linguistica (francese, wolof, inglese, arabo), piuttosto che le ripetizioni, le onomatopee o le interiezioni. L'effetto complessivo è, come segnala Mbaye Diouf, quello di un mosaico di lingue e di generi, in cui la sovrabbondanza suggerisce l'insufficienza, o, per meglio dire, l'incapacità di ogni lingua (e di tutte le lingue insieme) di dire l'esistente.

6 Motivazioni fortemente etiche sono alla base dell'opera letteraria di Ghania Hammadou, in cui si ritrova il legame tragico tra scrittura e sopravvivenza che ha caratterizzato la letteratura dell'emergenza in Algeria alla fine del secolo scorso. Scrivere per non morire o morire perché si scrive è il paradosso drammatico di fronte al quale si trova lo scrittore algerino durante la guerra civile che ha sconvolto il paese, al punto che i romanzi sono pervasi dal bisogno di «rendre compte du cauchemar vécu» (Christa Jones, p. 88) ma anche da una sostanziale «incapacité d'échapper à l'Histoire» (p. 93) che chiude ogni possibilità di vera rinascita.Conclude il volume un'interessante raccolta di annessi, in cui spiccano i foglietti autobiografici del Grand Cahier di Agota Kristof e alcuni inediti di Maria Maïlat.

Questo numero di «Francofonia» costituisce uno strumento importante di analisi della funzione della mobilità culturale nella scrittura femminile francofona. «Expatriées, exilées, voyageuses ou nomades, ces "femmes oiseaux" posent de nouvelles frontières et de nouveaux enjeux» (p. 13) che, come segnalano i curatori, arricchiscono i dibattiti letterari e spingono i critici a cercare nuovi orizzonti interpretativi. 